\title{
Relationship Between Nutritional Habits and Hair Calcium Levels in Young Women
}

\author{
Marta Jeruszka-Bielak • Anna Brzozowska
}

Received: 7 December 2010 / Accepted: 8 March 2011 /

Published online: 30 March 2011

(C) The Author(s) 2011. This article is published with open access at Springerlink.com

\begin{abstract}
The present study was conducted to investigate whether hair calcium levels are related to nutritional habits, selected status parameters, and life-style factors in young women. Eighty-five healthy female students neither pregnant nor lactating, using no hair dyes or permanents were recruited for the study. Food consumption data, including fortified products and dietary supplements were collected with 4-day records. The calcium levels in hair and serum were analyzed by atomic absorption spectroscopy. Serum osteocalcin and the C-terminal telopeptide of type I collagen were assayed by ELISA. The women were divided into four groups according to their total vitamin D and calcium intakes and hair calcium levels. At adequate calcium intake and comparable serum bone biomarker levels, supplemental vitamin D increased the hair calcium levels. On the other hand, at lower than estimated adequate requirement of vitamin D intake the hair calcium levels were comparable in women with low calcium intakes but consuming high amounts of meat products or those whose diets were rich in dairy products, possibly due to homeostatic mechanisms. Elevated hair calcium was seen in $25 \%$ of subjects and could not be related to nutritional or life-style factors. The results show that the hair calcium levels were weakly related to the quality of diet, with some synergistic interactions between nutrients, especially vitamin $\mathrm{D}$ and magnesium.
\end{abstract}

Keywords Calcium $\cdot$ Nutritional habits $\cdot$ Hair $\cdot$ Serum $\cdot$ Bone biomarkers

\section{Introduction}

The analysis of trace elements in hair has attracted the attention of scientists for several decades. The non-invasiveness of the method, ease of sample collection, and storage as well as advances in modern analytical techniques are some of the advantages of hair for determining the levels of various major, trace and toxic elements in animals and humans.

M. Jeruszka-Bielak $(\bowtie) \cdot$ A. Brzozowska

Department of Human Nutrition, Warsaw University of Life Sciences-SGGW, 159c Nowoursynowska

Str, 02-776 Warsaw, Poland

e-mail: marta_jeruszka_bielak@sggw.pl 
After minerals are incorporated into hair, they are no longer subject to the present metabolic condition reflecting the changes that have occurred over a reasonably long period of time. The limitations are due to the possibility of contamination from environmental sources, and to some degree, to the lack of reference values suitable for different minerals in different population groups [1-4].

The hair calcium $(\mathrm{Ca})$ concentration has been used as an indicator of disorders in $\mathrm{Ca}$ and bone metabolism [5] and as a predictor of risk of coronary heart disease [6]. It was also suggested that hair $\mathrm{Ca}$ and magnesium $(\mathrm{Mg})$ levels were more reliable indicators of spine bone mineral density than its concentration in serum in premenopausal women [7].

Studies on minerals concentration in hair have been conducted in various populations in the context of nutritional status [8-10] but only a few took into account, directly $[11,12]$ or indirectly $[6,13]$, food consumption patterns and the intake of nutrients, including minerals.

Consequently, the present study was conducted to investigate whether hair calcium levels in young women are related to nutritional habits and nutrient intake, also considering some biochemical parameters and life-style factors. This work is part of a larger research on the influence of an 8-week consumption of fortified foods on nutritional status in young women.

\section{Materials and Methods}

\section{Subjects}

The Bioethics Committee of the Polish National Food and Nutrition Institute approved the study protocol. Eighty-five female students aged 19-26 years were selected for the study. None was pregnant, lactating, using hair dyes or permanent treatments, or suffering from chronic diseases or taking any medications that could influence Ca metabolism. Their BMI was in the $16.2-29.4 \mathrm{~kg} \mathrm{~m}^{-2}$ range (median, $20.6 \mathrm{~kg} \mathrm{~m}^{-2}$ ). Information on hormonal contraceptive usage, smoking status, self-perceived physical activity, and health status were collected by means of a questionnaire.

\section{Methods}

\section{Food and Nutrient Intake}

The energy and nutrient intakes were calculated with the "Nutrition" software from the Department of Human Nutrition, Warsaw, Poland.

Four-day records (four consecutive days including one weekend day) were used to assess the food and water intake. The 4-day records were collected during the base-line period (habitual intake), during the "washout" period without fortified products and dietary supplements and during the intervention study. Using food portions photographs and food labels, a trained interviewer supervised the portions of the foods consumed by the participants [14]. Data on consumption of fortified foods or dietary supplements were collected by means of a questionnaire and photo album of such products [15].

The medians of within-personal variations in macronutrient intakes in three periods of the study ranged between $11.2 \%$ and $16.0 \%$, being lowest for energy and highest for fiber. The variations were greater for micronutrients: $20.3 \%$ for Ca, $24.1 \%$ for vitamin D and especially higher for vitamins $\mathrm{C}(33.9 \%)$ and A $(32.7 \%)$. It should be remembered that the data were obtained at a normal nutrition period and during the washout and intervention periods. Herein, the data from the base-line period are shown. 


\section{Hair and Serum Analysis}

Hair samples ( $3 \mathrm{~cm}$ from skin) were collected from six different points from the neck. The samples were cut and washed according to IAEA method, rinsed in acetone (Merck1.00014.1000, Germany), three times in ultrapure water, and again in acetone. After drying to constant mass, samples were digested with $60 \% \mathrm{HNO}_{3}$ (Merck 1.00456.1000; Germany) in a microwave cavity (MARS 5, CEM, USA). Calcium was measured by AAS (UNICAM 989, SOLAAR, UK) after dilution with 5\% CsCl solution using a nitrous oxide-acetylene flame.

Venous blood samples were taken mornings in fasting state. Calcium in serum was measured by AAS, after microwave digestion similar to hair samples.

Reference materials for $\mathrm{Ca}$ were obtained from IAEA-085 (Austria) for hair and Seronorm $^{\mathrm{TM}}$ Trace Elements Serum L-1 (Norway) for serum. Recovery for hair was $98.6 \%$ while for serum it was $94.8 \%$. The coefficients of variation were $6.0 \%$ for hair and $0.75 \%$ for serum.

Serum osteocalcin (OC; biomarker of bone formation) and C-terminal telopeptide of type I collagen (CTx; biomarker of bone resorption) were assayed with a immuno-enzymatic ELISA kit from Nordic Bioscience Diagnostics, Denmark; Microplate Reader Pointe, USA.

\section{Statistical Analysis}

The Statistica PL v.8.0 computer program was used for all statistical analysis. The data were tested for distribution with the Shapiro-Wilk test. The data were log-transformed, when necessary. For descriptive purposes, the results are presented using untransformed values as means \pm standard deviation as well as median and range.

Cluster analysis was conducted to discriminate subjects with different nutritional status and/or nutrient intake. Before clustering, all variables such as serum OC, CTx, and $\mathrm{Ca}$ concentrations; hair $\mathrm{Ca}$ level; energy, total protein, animal protein, total $\mathrm{Ca}$, and vitamin $\mathrm{D}$ from normal diet; $\mathrm{Ca}$ and vitamin $\mathrm{D}$ from fortified and non-fortified food products; $\mathrm{Ca}$ and vitamin D from supplements; the intake of other nutrients as well as the intake of food groups were standardized in order to eliminate differences in their magnitudes.

The K-means procedure was applied which is based on Euclidean distance of objects from the cluster center in an iterative manner. Firstly, the data set is scanned for initial cluster centers and then the algorithm moves the objects between clusters and new cluster centers are calculated. This procedure is repeated until the distances between the objects within a cluster are smaller than the distances between the cluster centers. Because the number of clusters need to be selected a priori, separate analyses were performed using two, three, four, five, and six clusters. Also, the decision about the number and type of applied variables was made after conducting a number of analyses. Significantly, most homogenous groups (minimalized within-group variation) with the highest differences between each other (maximized between-group variation) were achieved when 4 clusters were separated and three variables were used: calcium level in hair, total intake of $\mathrm{Ca}$ and total intake of vitamin D (ANOVA, $F$ test: 46.2; 41.7 and 103, respectively, and $p<0.0001$ for each). Clusters can be described as "Low $\mathrm{Ca}$ intake, low hair Ca" (I), "Low Ca intake, elevated hair Ca" (II), "Very high intake of vitamin D, high hair Ca" (III) and "High Ca intake, low hair Ca" (IV). Individual characteristics in clusters were compared using Student's $t$ test and Chi-square test for quantitative and qualitative data, respectively.

The Pearson's correlation was used to examine the relationship between quantitative variables. Besides, a stepwise multiple regression was applied in order to determine the 
factors that influenced hair calcium level. For all analysis, $p$ values $<0.05$ were considered statistically significant.

\section{Results}

The hair calcium level, total $\mathrm{Ca}$, and vitamin $\mathrm{D}$ intake in clusters are presented in Table 1. Women in cluster I ( $49 \%$ of subjects) had a significantly lower mean Ca intake than those in other clusters and lower hair Ca level than in clusters II and III. In cluster IV (16\% of subjects), vitamin D intake and hair Ca were similar to cluster I, but the Ca intake was significantly higher. Elevated hair Ca level (above reference values) was found in cluster II ( $25 \%$ of subjects), in which both nutrients intake was quite low.

Cluster III ( $9 \%$ of subjects) had more than five times higher vitamin D intake and also higher calcium intake and level in hair than other subgroups. Further analysis showed that almost all women in cluster III (seven out of eight) took dietary supplements with vitamin $\mathrm{D}$, while in other clusters there was either no subjects (cluster II and IV) or a very small number of subjects (three out of 42) taking such supplements. Also Ca supplements were significantly more popular in cluster III ( $50 \%$ of subjects) than in other subgroups $(7.0 \%$, $14.3 \%$, and $0 \%$, respectively in clusters I, II, and IV).

Although clusters did not differ significantly in their health and life-style factors (Table 2), it is worth noticing that women in clusters III and IV had a more positive perception of their health and physical activity than women in clusters I and II. Also, there were no smokers in cluster III, while there were 14, three, and three smokers in clusters I, II, and IV, respectively.

Table 1 Hair calcium level, total calcium and vitamin D intakes of women in all clusters

\begin{tabular}{|c|c|c|c|c|c|}
\hline & \multirow[t]{2}{*}{ Reference values } & \multicolumn{4}{|l|}{ Cluster } \\
\hline & & I $(n=42)$ & II $(n=21)$ & III $(n=8)$ & IV $(n=14)$ \\
\hline \multicolumn{6}{|c|}{ Hair Ca ( $\mu \mathrm{g} / \mathrm{g}$ dry matter) } \\
\hline $\mathrm{X} \pm \mathrm{SD}$ & & $650 \pm 248 \mathrm{a}$ & $1,651 \pm 464 \mathrm{~b}$ & $1,002 \pm 383 \mathrm{c}$ & $682 \pm 275 \mathrm{a}, \mathrm{c}$ \\
\hline Median & & 610 & 1,447 & 1,113 & 575 \\
\hline Range & $400-1,000^{\mathrm{a}}$ & $270-1,154$ & $1,150-2,505$ & $360-1,409$ & $401-1,373$ \\
\hline \multicolumn{6}{|c|}{ Total Ca intake (mg/day) } \\
\hline $\mathrm{X} \pm \mathrm{SD}$ & & $617 \pm 137 \mathrm{a}$ & $745 \pm 161 \mathrm{~b}$ & $1,011 \pm 151 \mathrm{c}$ & $1,108 \pm 201 \mathrm{c}$ \\
\hline Median & $800^{\mathrm{b}}$ & 661 & 733 & 1,049 & 1,077 \\
\hline Range & & $338-837$ & $484-1,207$ & $823-1,183$ & $904-1,578$ \\
\hline \multicolumn{6}{|c|}{ Total vitamin $D$ intake ( $\mu \mathrm{g} /$ day) } \\
\hline $\mathrm{X} \pm \mathrm{SD}$ & & $3.2 \pm 2.0 \mathrm{a}$ & $2.1 \pm 1.2 \mathrm{~b}$ & $14.6 \pm 2.1 \mathrm{c}$ & $2.98 \pm 1.76 \mathrm{a}, \mathrm{b}$ \\
\hline Median & $10^{\mathrm{b}}$ & 2.5 & 2.0 & 14.7 & 2.45 \\
\hline Range & & $0.7-9.3$ & $0.5-5.2$ & $11.4-18.7$ & $1.1-6.7$ \\
\hline
\end{tabular}

Values in rows not sharing a common letter are significantly different $(p<0.05)$

$X \pm S D$ arithmetic mean \pm standard deviation

${ }^{\text {a }}$ Reference values for Polish population [16]

${ }^{\mathrm{b}}$ Estimated average requirements (EAR) for calcium and vitamin D for women aged 19-30 years according to IOM [17] 
Table 2 Health and life-style factors for women in all clusters

\begin{tabular}{|c|c|c|c|c|}
\hline & \multicolumn{4}{|l|}{ Cluster } \\
\hline & $\begin{array}{l}\text { I }(n=42) \\
n(\%)\end{array}$ & $\begin{array}{l}\text { II }(n=21) \\
n(\%)\end{array}$ & $\begin{array}{l}\text { III }(n=8) \\
n(\%)\end{array}$ & $\begin{array}{l}\text { IV }(n=14) \\
n(\%)\end{array}$ \\
\hline \multicolumn{5}{|l|}{ Self-perceived health status ${ }^{a}$} \\
\hline Very good & $8(19.0)$ & $3(14.3)$ & $1(12.5)$ & $5(35.7)$ \\
\hline Good & $23(54.8)$ & $12(57.1)$ & $7(87.5)$ & $9(64.3)$ \\
\hline Moderate & $10(23.8)$ & $2(9.5)$ & 0 & 0 \\
\hline \multicolumn{5}{|l|}{ Self-perceived physical activity ${ }^{a}$} \\
\hline High & $3(7.1)$ & $2(9.5)$ & 0 & $2(14.3)$ \\
\hline Moderate & $28(66.7)$ & $11(52.4)$ & $8(100)$ & $10(71.4)$ \\
\hline Low & $8(19.0)$ & $8(38.1)$ & 0 & $1(7.1)$ \\
\hline \multicolumn{5}{|l|}{ BMI } \\
\hline Underweight $\left(<18.5 \mathrm{~kg} / \mathrm{m}^{2}\right)$ & $4(9.5)$ & $4(19.0)$ & $2(25.0)$ & $1(7.1)$ \\
\hline Normal Range(18.5-24.9 kg/m²) & $34(80.9)$ & $16(76.2)$ & $6(75.0)$ & $13(92.9)$ \\
\hline Overweight $\left(25.0-29.9 \mathrm{~kg} / \mathrm{m}^{2}\right)$ & $4(9.5)$ & $1(4.8)$ & 0 & 0 \\
\hline Hormonal contraceptive usage & $8(19.1)$ & $3(14.3)$ & $2(25.0)$ & $2(14.3)$ \\
\hline Smoking & $14(33.3)$ & $3(14.3)$ & 0 & $3(21.4)$ \\
\hline
\end{tabular}

${ }^{\text {a }}$ Percentages in some cases do not sum up to $100 \%$ as some respondents did not evaluate their health or physical activity status

The intakes of some foods, macro- and micronutrients are presented in Tables 3, 4, and 5. In general, these were lower in clusters I and II compared with cluster IV and particularly to cluster III.

Considering the energy content in the diets, women in clusters I and II could be classified as "small eaters" but they differed significantly in meat, fish and poultry consumption and as a consequence in dietary $\mathrm{Ca} / \mathrm{P}$ ratio. Cluster I can be described as the "meat eaters". On the basis of food groups women in cluster III can be classified as "cereal eaters" and "supplement users", while those in cluster IV as "dairy products eaters". For women in cluster IV, food products (including water) were the only source of $\mathrm{Ca}$, while in cluster III supplements were the important source. The dietary $\mathrm{Ca} / \mathrm{P}$ ratio was more consistent in clusters III and IV than in cluster I. There were no differences in consumption of eggs, butter and cream among clusters.

No significant differences were found among clusters in relation to serum biomarkers, with the exception for serum $\mathrm{Ca}$ in cluster II, which was lower to that of cluster IV (Table 6). The highest serum OC and CTx were seen in cluster III.

To assess the relationship between hair calcium and intake of nutrients or serum levels, the Pearson's correlation was applied to the whole population, or to subpopulations such as a group of women with hair $\mathrm{Ca}$ level above or within reference values. The significant correlations found in cluster IV are given in the "Discussion" section.

Also, stepwise multiple regressions were used with hair $\mathrm{Ca}$ level as a single dependent variable to look for variables that might explain its accumulation in hair. After conducting a number of analyses (also in the whole population and in subpopulations), it turned out that the best possible model included only one variable, i.e., total intake of magnesium: higher intake of $\mathrm{Mg}$ was connected with higher hair Ca level, but its $R^{2}=18.9 \%$, meaning that only $19 \%$ of 
Table 3 Intakes of some food products

\begin{tabular}{|c|c|c|c|c|}
\hline \multirow[t]{2}{*}{ Intake (g/day) } & \multicolumn{4}{|l|}{ Cluster } \\
\hline & $\mathrm{I}(n=42)$ & II $(n=21)$ & III $(n=8)$ & IV $(n=14)$ \\
\hline \multicolumn{5}{|c|}{ Milk and dairy products } \\
\hline $\mathrm{X} \pm \mathrm{SD}$ & $510 \pm 246$ a & $595 \pm 251 \mathrm{a}, \mathrm{b}$ & $804 \pm 300 \mathrm{~b}$ & $1,017 \pm 252^{\mathrm{c}}$ \\
\hline Median & 502 & 591 & 798 & 973 \\
\hline Range & $151-1,502$ & $156-1,332$ & $467-1,406$ & $652-1,612$ \\
\hline \multicolumn{5}{|l|}{ Cereals } \\
\hline $\mathrm{X} \pm \mathrm{SD}$ & $139 \pm 36 \mathrm{a}$ & $128 \pm 44 \mathrm{a}$ & $179 \pm 60 \mathrm{~b}$ & $155 \pm 42 \mathrm{a}, \mathrm{b}$ \\
\hline Median & 140 & 124 & 183 & 167 \\
\hline Range & $61-209$ & $60-226$ & $89-262$ & $28-201$ \\
\hline \multicolumn{5}{|l|}{ Eggs } \\
\hline $\mathrm{X} \pm \mathrm{SD}$ & $20 \pm 14$ & $20 \pm 17$ & $24 \pm 20$ & $26 \pm 15$ \\
\hline Median & 21 & 15 & 14 & 28 \\
\hline Range & $0-45$ & $0-58$ & $3-64$ & $2-47$ \\
\hline \multicolumn{5}{|c|}{ Meat, fish, and poultry } \\
\hline $\mathrm{X} \pm \mathrm{SD}$ & $141 \pm 62 \mathrm{a}$ & $98 \pm 48 \mathrm{~b}$ & $121 \pm 83 \mathrm{a}, \mathrm{b}$ & $80 \pm 47 b$ \\
\hline Median & 141 & 99 & 123 & 75 \\
\hline Range & $37-280$ & $12-210$ & $0-223$ & $7-186$ \\
\hline \multicolumn{5}{|c|}{ Butter and cream } \\
\hline $\mathrm{X} \pm \mathrm{SD}$ & $13 \pm 7$ & $10 \pm 9$ & $19 \pm 15$ & $15 \pm 11$ \\
\hline Median & 12 & 8 & 14 & 12 \\
\hline Range & $0-29$ & $0-34$ & $4-46$ & $1-34$ \\
\hline \multicolumn{5}{|c|}{ Fruit and vegetables } \\
\hline $\mathrm{X} \pm \mathrm{SD}$ & $584 \pm 329$ & $727 \pm 496$ & $615 \pm 388$ & $681 \pm 385$ \\
\hline Median & 554 & 585 & 588 & 600 \\
\hline Range & $64-1,990$ & $123-2,210$ & $128-1,260$ & $215-1,540$ \\
\hline \multicolumn{5}{|l|}{ Coffee $^{a}$} \\
\hline $\mathrm{X} \pm \mathrm{SD}$ & $92 \pm 112$ & $139 \pm 165$ & $106 \pm 124$ & $145 \pm 166$ \\
\hline Median & 50 & 81 & 62 & 134 \\
\hline Range & $0-375$ & $0-560$ & $0-300$ & $0-610$ \\
\hline
\end{tabular}

Values in rows not sharing a common letter are significantly different $(p<0.05)$

$X \pm S D$ arithmetic mean \pm standard deviation

${ }^{\text {a }}$ In milliliters

variation in $\mathrm{Ca}$ level in hair could be explained by the total intake of magnesium and over $80 \%$ remained unexplained.

\section{Discussion}

Calcium bioavailability, metabolism, and status are influenced not just by the total calcium intake but also by vitamin D and other factors like the presence of promoters or inhibitors of its absorption in the food consumed, additional host-related factors such as overall 
Table 4 Intakes of macronutrients

\begin{tabular}{|c|c|c|c|c|c|}
\hline \multirow[t]{2}{*}{ Intakes } & \multirow[t]{2}{*}{ Reference values } & \multicolumn{4}{|l|}{ Cluster } \\
\hline & & $\mathrm{I}(n=42)$ & II $(n=21)$ & III $(n=8)$ & IV $(n=14)$ \\
\hline \multicolumn{6}{|c|}{ Energy (kcal) } \\
\hline $\mathrm{X} \pm \mathrm{SD}$ & & $1,584 \pm 286 \mathrm{a}$ & $1,518 \pm 362 \mathrm{a}, \mathrm{b}$ & $1,854 \pm 488 \mathrm{~b}, \mathrm{c}$ & $1,858 \pm 290 \mathrm{c}$ \\
\hline Median & $1,900-2400^{\mathrm{a}}$ & 1,600 & 1,570 & 1,938 & 1,810 \\
\hline Range & & $993-2,116$ & $787-2,024$ & $944-2,390$ & $1,480-2,339$ \\
\hline \multicolumn{6}{|c|}{ Protein $(\mathrm{g})$} \\
\hline $\mathrm{X} \pm \mathrm{SD}$ & & $57.7 \pm 9.3 \mathrm{a}$ & $55.3 \pm 12.9 \mathrm{a}$ & $67.0 \pm 13.4 \mathrm{~b}$ & $68.6 \pm 9.0 \mathrm{~b}$ \\
\hline Median & $33-58^{\mathrm{b}}$ & 57 & 55 & 69 & 69 \\
\hline Range & & $42-80$ & $36-77$ & $46-85$ & $57-89$ \\
\hline \multicolumn{6}{|l|}{ Fat $(g)$} \\
\hline $\mathrm{X} \pm \mathrm{SD}$ & & $61.5 \pm 17.2 \mathrm{a}$ & $60.5 \pm 19.5 \mathrm{a}$ & $77.4 \pm 17.3 \mathrm{~b}$ & $77.1 \pm 18.1 \mathrm{~b}$ \\
\hline Median & $51-98^{\mathrm{b}}$ & 60 & 57 & 75 & 71 \\
\hline Range & & $26-96$ & $21-95$ & $46-100$ & $57-111$ \\
\hline \multicolumn{6}{|c|}{ Carbohydrates (g; total) } \\
\hline $\mathrm{X} \pm \mathrm{SD}$ & & $214 \pm 44 \mathrm{a}, \mathrm{b}$ & $202 \pm 56 \mathrm{a}$ & $237 \pm 77 \mathrm{a}, \mathrm{b}$ & $241 \pm 36 b$ \\
\hline Median & $260-330^{\mathrm{c}}$ & 210 & 193 & 261 & 241 \\
\hline Range & & $130-310$ & $104-313$ & $93-312$ & $183-300$ \\
\hline \multicolumn{6}{|l|}{ Fiber $(\mathrm{g})$} \\
\hline $\mathrm{X} \pm \mathrm{SD}$ & & $16.1 \pm 4.2 \mathrm{a}$ & $17.5 \pm 6.6 \mathrm{a}, \mathrm{b}$ & $16.1 \pm 5.6 \mathrm{a}, \mathrm{b}$ & $19.5 \pm 5.1 \mathrm{~b}$ \\
\hline Median & $20-40^{\mathrm{d}}$ & 15.9 & 17.7 & 18.1 & 18.9 \\
\hline Range & & $8.7-29.5$ & $7.8-32.0$ & $6.9-23.1$ & $12.8-30.1$ \\
\hline
\end{tabular}

Values in rows not sharing a common letter are significantly different $(p<0.05)$

$X \pm S D$ arithmetic mean \pm standard deviation

${ }^{\text {a }}$ EER for Polish women aged 19-30 years, low to moderate physical activity level [18]

${ }^{\mathrm{b}}$ EAR for Polish women aged 19-30 years old [18]

${ }^{\mathrm{c}}$ Assumption: at least 55\% of energy should come from carbohydrates [18]

${ }^{\mathrm{d}}$ Recommendations for Polish adults [18]

health, hormonal status, physical activity, and coexisting diseases as well as the use of medications that might affect $\mathrm{Ca}$ absorption [20].

As a consequence, the levels of $\mathrm{Ca}$ in organs, tissues and hair are the result of several variables. In addition, vitamin D requires sunlight for its biosynthesis, so geographical location and climatic conditions play additional decisive roles in Ca metabolism. Because our study started in February and participants were students living in the same general area, it can be assumed that they had similar exposure to sunlight for at least 6 months.

In light of recently revised Institute of Medicine Dietary Reference Intakes for vitamin D [17], the only subgroup in our study with intake (mainly from supplements) higher than estimated average requirements (EAR), was cluster III. The dietary intake of this nutrient among the rest of the participants was very low.

It could be assumed that adequate or above EARs intake of vitamin D and Ca observed in cluster III would result in higher intracellular pool calcium and in turn, higher amounts of $\mathrm{Ca}$ in hair. This was not the case in cluster IV. Although in both clusters III and IV the total intake of $\mathrm{Ca}$ and most other nutrients was about the same, with comparable life-style 
Table 5 Intakes of some micronutrients

\begin{tabular}{|c|c|c|c|c|c|}
\hline \multirow[t]{2}{*}{ Intake } & \multirow[t]{2}{*}{ Reference values } & \multicolumn{4}{|l|}{ Cluster } \\
\hline & & $\mathrm{I}(n=42)$ & II $(n=21)$ & III $(n=8)$ & IV $(n=14)$ \\
\hline \multicolumn{6}{|l|}{$\mathrm{Mg}(\mathrm{mg})$} \\
\hline $\mathrm{X} \pm \mathrm{SD}$ & & $277 \pm 75 \mathrm{a}$ & $319 \pm 117 \mathrm{a}, \mathrm{b}$ & $337 \pm 78 \mathrm{~b}$ & $348 \pm 67 \mathrm{~b}$ \\
\hline Median & $255^{\mathrm{a}}$ & 263 & 296 & 344 & 348 \\
\hline Range & & $114-506$ & $153-644$ & $223-462$ & $246-468$ \\
\hline \multicolumn{6}{|l|}{$\mathrm{K}(\mathrm{mg})$} \\
\hline $\mathrm{X} \pm \mathrm{SD}$ & & $2,671 \pm 55 \mathrm{a}$ & $2,757 \pm 772 \mathrm{a}$ & $2,685 \pm 791 \mathrm{a}, \mathrm{b}$ & $3,180 \pm 558 \mathrm{~b}$ \\
\hline Median & $4,700^{\mathrm{b}}$ & 2,614 & 2,692 & 2,678 & 3,033 \\
\hline Range & & $1,022-3,877$ & $1,440-4,370$ & $1,354-3,817$ & $2,594-4,465$ \\
\hline \multicolumn{6}{|l|}{$\mathrm{Na}^{\mathrm{c}}(\mathrm{mg})$} \\
\hline $\mathrm{X} \pm \mathrm{SD}$ & & $2,403 \pm 1,326$ & $2,029 \pm 688$ & $2,575 \pm 691$ & $2,053 \pm 777$ \\
\hline Median & $1,500^{\mathrm{b}}$ & 2,007 & 1,922 & 2,708 & 2,482 \\
\hline Range & & $691-6,954$ & $1,034-3,809$ & $1,543-3,393$ & $1,196-4,234$ \\
\hline \multicolumn{6}{|l|}{$\mathrm{P}(\mathrm{mg})$} \\
\hline $\mathrm{X} \pm \mathrm{SD}$ & & $1,100 \pm 160 \mathrm{a}$ & $1,130 \pm 231 \mathrm{a}, \mathrm{b}$ & $1,341 \pm 247 \mathrm{~b}, \mathrm{c}$ & $1,511 \pm 215 \mathrm{c}$ \\
\hline Median & $580^{\mathrm{a}}$ & 1,074 & 1,110 & 1,332 & 1,463 \\
\hline Range & & $718-1,486$ & $696-1,440$ & $1,005-1,756$ & $1,214-2,059$ \\
\hline \multicolumn{6}{|c|}{$\mathrm{Ca} / \mathrm{P}$ in $\operatorname{diet}(\mathrm{mg} / \mathrm{mg})$} \\
\hline $\mathrm{X} \pm \mathrm{SD}$ & & $0.57 \pm 0.11 \mathrm{a}$ & $0.66 \pm 0.17 \mathrm{~b}$ & $0.78 \pm 0.18 \mathrm{~b}$ & $0.74 \pm 0.08 \mathrm{~b}$ \\
\hline Median & $>1$ & 0.57 & 0.65 & 0.82 & 0.73 \\
\hline Range & & $0.38-0.80$ & $0.34-1.10$ & $0.53-1.04$ & $0.65-0.96$ \\
\hline \multicolumn{6}{|l|}{$\mathrm{Fe}(\mathrm{mg})$} \\
\hline $\mathrm{X} \pm \mathrm{SD}$ & & $11.5 \pm 6.1 \mathrm{a}$ & $10.7 \pm 4.6 \mathrm{a}$ & $19.5 \pm 11.1 \mathrm{~b}$ & $11.7 \pm 2.21 \mathrm{a}$ \\
\hline Median & $8^{\mathrm{a}}$ & 9.38 & 10.6 & 16.6 & 12.0 \\
\hline Range & & $6.40-31.1$ & $5.00-25.8$ & $8.70-41.7$ & $8.00-15.7$ \\
\hline \multicolumn{6}{|l|}{$\mathrm{Zn}(\mathrm{mg})$} \\
\hline $\mathrm{X} \pm \mathrm{SD}$ & & $11.5 \pm 10.5$ & $11.8 \pm 6.8$ & $13.3 \pm 5.80$ & $14.6 \pm 10.7$ \\
\hline Median & $6.8^{\mathrm{a}}$ & 8.30 & 10.1 & 10.6 & 10.5 \\
\hline Range & & $4.91-62.6$ & $4.80-29.5$ & $7.70-22.5$ & $7.60-48.3$ \\
\hline \multicolumn{6}{|l|}{$\mathrm{Cu}(\mathrm{mg})$} \\
\hline $\mathrm{X} \pm \mathrm{SD}$ & & $1.15 \pm 0.70 \mathrm{a}$ & $1.26 \pm 0.83 \mathrm{a}, \mathrm{b}$ & $1.52 \pm 0.76 \mathrm{a}, \mathrm{b}$ & $1.50 \pm 0.73 \mathrm{~b}$ \\
\hline Median & $0.7^{\mathrm{a}}$ & 0.98 & 1.1 & 1.20 & 1.30 \\
\hline Range & & $0.50-3.90$ & $0.50-4.00$ & $0.89-2.90$ & $1.00-3.90$ \\
\hline \multicolumn{6}{|c|}{ Vitamin A $(\mu g)$} \\
\hline $\mathrm{X} \pm \mathrm{SD}$ & & $1,143 \pm 811 \mathrm{a}$ & $1,478 \pm 1353 \mathrm{a}, \mathrm{b}$ & $1,553 \pm 412 \mathrm{~b}$ & $1,577 \pm 991 \mathrm{a}, \mathrm{b}$ \\
\hline Median & $500^{\mathrm{a}}$ & 876 & 1,125 & 1,674 & 1,147 \\
\hline Range & & $293-3,924$ & $212-5,916$ & $916-2,017$ & $381-3,315$ \\
\hline \multicolumn{6}{|c|}{ Vitamin E (mg) } \\
\hline $\mathrm{X} \pm \mathrm{SD}$ & & $19.9 \pm 35.5$ & $15.9 \pm 13.8$ & $18.4 \pm 6.8$ & $25.1 \pm 30.8$ \\
\hline Median & $8^{\mathrm{b}}$ & 9.6 & 12.0 & 17.5 & 14.6 \\
\hline Range & & $4.60-215$ & $3.35-69.4$ & $8.9-26.3$ & $9.30-123$ \\
\hline \multicolumn{6}{|c|}{ Vitamin C (mg) } \\
\hline $\mathrm{X} \pm \mathrm{SD}$ & & $88.2 \pm 112$ & $101 \pm 81.0$ & $108 \pm 26.7$ & $85.5-32.6$ \\
\hline
\end{tabular}


Table 5 (continued)

\begin{tabular}{|c|c|c|c|c|c|}
\hline \multirow[t]{2}{*}{ Intake } & \multirow[t]{2}{*}{ Reference values } & \multicolumn{4}{|l|}{ Cluster } \\
\hline & & I $(n=42)$ & II $(n=21)$ & III $(n=8)$ & IV $(n=14)$ \\
\hline Median & $60^{\mathrm{a}}$ & 56.0 & 84.1 & 99.1 & 89.0 \\
\hline Range & & $9.60-725$ & $20.7-369$ & $77.5-151$ & $19.3-141$ \\
\hline \multicolumn{6}{|c|}{ Vitamin $B_{1}(\mathrm{mg})$} \\
\hline $\mathrm{X} \pm \mathrm{SD}$ & & $1.47 \pm 1.03 \mathrm{a}$ & $1.25 \pm 1.08 \mathrm{a}$ & $3.33 \pm 1.88 \mathrm{~b}$ & $1.14 \pm 0.37 \mathrm{a}$ \\
\hline Median & $0.9^{\mathrm{a}}$ & 1.01 & 1.02 & 2.34 & 1.09 \\
\hline Range & & $0.54-4.70$ & $0.44-5.40$ & $1.63-6.18$ & $0.68-2.01$ \\
\hline \multicolumn{6}{|c|}{ Vitamin $B_{2}(\mathrm{mg})$} \\
\hline $\mathrm{X} \pm \mathrm{SD}$ & & $1.86 \pm 1.20 \mathrm{a}$ & $1.57 \pm 0.57 \mathrm{a}$ & $4.33 \pm 3.11 \mathrm{~b}$ & $2.70 \pm 2.94 \mathrm{a}$ \\
\hline Median & $0.9^{\mathrm{a}}$ & 1.31 & 1.31 & 3.50 & 1.79 \\
\hline Range & & $0.87-5.53$ & $0.98-2.85$ & $2.24-11.8$ & $1.39-12.7$ \\
\hline \multicolumn{6}{|c|}{ Vitamin $\mathrm{B}_{6}(\mathrm{mg})$} \\
\hline $\mathrm{X} \pm \mathrm{SD}$ & & $2.47 \pm 1.58 \mathrm{a}$ & $3.07 \pm 4.19 \mathrm{a}$ & $5.77 \pm 5.66 \mathrm{~b}$ & $2.99 \pm 2.84 \mathrm{a}, \mathrm{b}$ \\
\hline Median & $1.1^{\mathrm{a}}$ & 1.83 & 1.94 & 3.06 & 2.26 \\
\hline Range & & $0.81-6.68$ & $0.80-23.83$ & $1.98-16.6$ & $1.18-12.6$ \\
\hline \multicolumn{6}{|c|}{ Vitamin PP (mg) } \\
\hline $\mathrm{X} \pm \mathrm{SD}$ & & $22.2 \pm 17.0 \mathrm{a}$ & $14.8 \pm 8.9 \mathrm{~b}$ & $25.8 \pm 8.0 \mathrm{a}$ & $21.7 \pm 28.2 \mathrm{a}, \mathrm{b}$ \\
\hline Median & $11^{\mathrm{a}}$ & 16.9 & 13.8 & 26.3 & 14.6 \\
\hline Range & & $8.80-108$ & $6.40-48.1$ & $12.5-35.5$ & $6.30-119$ \\
\hline \multicolumn{6}{|c|}{ Folates $(\mu \mathrm{g})$} \\
\hline $\mathrm{X} \pm \mathrm{SD}$ & & $220 \pm 120 \mathrm{a}, \mathrm{b}$ & $199 \pm 68$ a & $306 \pm 158 \mathrm{~b}$ & $257 \pm 105 \mathrm{a}, \mathrm{b}$ \\
\hline Median & $320^{\mathrm{a}}$ & 184 & 193 & 241 & 245 \\
\hline Range & & $117-666$ & $86.3-355$ & $134-538$ & $121-563$ \\
\hline \multicolumn{6}{|c|}{ Vitamin $B_{12}(\mu g)$} \\
\hline $\mathrm{X} \pm \mathrm{SD}$ & & $4.16 \pm 2.29 \mathrm{a}$ & $3.61 \pm 2.00 \mathrm{a}$ & $7.47 \pm 3.27 \mathrm{~b}$ & $6.09 \pm 3.52 \mathrm{~b}$ \\
\hline Median & $2.0^{\mathrm{a}}$ & 3.11 & 2.87 & 8.12 & 4.45 \\
\hline Range & & $1.70-8.80$ & $1.39-8.08$ & $3.46-12.5$ & $3.10-14.1$ \\
\hline
\end{tabular}

Values in rows not sharing a common letter are significantly different $(p<0.05)$

$X \pm S D$ arithmetic mean \pm standard deviation

${ }^{\text {a }}$ EAR for Polish women aged 19-30 years [18]

${ }^{\mathrm{b}}$ AI for Polish women aged 19-30 years [18]

${ }^{\mathrm{c}}$ Sodium derived from table salt was not calculated

factors, a low vitamin D level in cluster IV was probably the main reason of almost two times lower hair calcium level in this subgroup than in cluster III. For example, men supplemented with vitamin D plus antioxidants for 4 months resulted in larger Ca levels in their beard [21]. MacPerson and Bacsó in a study involving more than 4,000 men living in different regions of the UK found direct relationships between hair $\mathrm{Ca}$ levels and annual sunshine hours and hardness of tap water [6].

The main consequences of vitamin D deficiency are impairment of intestinal $\mathrm{Ca}$ absorption and a series of adaptive responses or compensatory actions. Firstly, decreased serum $\mathrm{Ca}^{2+}$ level stimulates parathyroid hormone (PTH) secretion, which in turn promotes the production of an active metabolite of vitamin $\mathrm{D}\left(1,25-(\mathrm{OH})_{2} \mathrm{D}\right)$ and thus increases 
Table 6 Serum calcium (Ca), osteocalcin (OC), and CTx concentrations

\begin{tabular}{|c|c|c|c|c|c|}
\hline & \multirow[t]{2}{*}{ Reference values } & \multicolumn{4}{|l|}{ Cluster } \\
\hline & & $\mathrm{I}(n=42)$ & II $(n=21)$ & III $(n=8)$ & $\operatorname{IV}(n=14)$ \\
\hline \multicolumn{6}{|c|}{ Serum Ca (mg/l) } \\
\hline $\mathrm{X} \pm \mathrm{SD}$ & & $102 \pm 6.3 \mathrm{a}, \mathrm{b}$ & $99.7 \pm 9.0 \mathrm{a}$ & $104 \pm 6.4 \mathrm{a}, \mathrm{b}$ & $106 \pm 5.7 \mathrm{~b}$ \\
\hline Median & & 103 & 99.5 & 105 & 105 \\
\hline Range & $85-105^{\mathrm{a}}$ & $86.6-116$ & $82.1-115$ & $91.1-111$ & $97.4-116$ \\
\hline \multicolumn{6}{|c|}{ Serum OC (ng/ml) } \\
\hline $\mathrm{X} \pm \mathrm{SD}$ & $17.9 \pm 6.5^{b}$ & $26.8 \pm 10.9$ & $30.0 \pm 16.3$ & $34.6 \pm 15.8$ & $25.2 \pm 9.3$ \\
\hline Median & & 23.8 & 25.3 & 32.6 & 23.6 \\
\hline Range & & $12.1-57.2$ & $9.9-71.6$ & $9.6-63.8$ & $9.7-46.1$ \\
\hline \multicolumn{6}{|c|}{ Serum CTx (ng/ml) } \\
\hline $\mathrm{X} \pm \mathrm{SD}$ & & $0.71 \pm 0.28$ & $0.76 \pm 0.45$ & $0.93 \pm 0.37$ & $0.81 \pm 0.26$ \\
\hline Median & 0.287 & 0.69 & 0.66 & 0.82 & 0.76 \\
\hline Range & $0.112-0.738^{\mathrm{c}}$ & $0.23-1.77$ & $0.10-1.90$ & $0.50-1.64$ & $0.38-1.25$ \\
\hline
\end{tabular}

Values in rows not sharing a common letter are significantly different $(p<0.05)$

$X \pm S D$ arithmetic mean \pm standard deviation

${ }^{\text {a }}$ From reference [19]

${ }^{\mathrm{b}}$ Reference values for premenopausal women (kit leaflet)

${ }^{\mathrm{c}}$ Reference values for premenopausal women (geometric mean and 95\% CI; kit leaflet)

intestinal $\mathrm{Ca}$ absorption. In addition to this partial adaptation, $\mathrm{PTH}$ promotes renal $\mathrm{Ca}$ reabsorption, a positive compensation, but accelerates bone resorption, a negative compensatory action on bone health [22]. When normocalcemia is achieved as a result of these actions of PTH there is no extra Ca pool for deposition in hair (cluster IV).

The interpretation of bone biomarkers values in young women is somewhat complicated due to lack of reference values for the 19-25 age group. For women in the 20-29 age segment, higher levels of bone formation and bone resorption markers reflect bone consolidation process occurring in this age range, just the opposite to what occurs in elderly women [23]. In our study, no significant differences in bone biomarkers were observed among clusters, but the serum OC and CTx values were slightly higher in cluster III than in the other subgroups.

It is worth noting that although the intake of $\mathrm{Ca}$ and other nutrients beside vitamin $\mathrm{D}$ are higher in women in cluster IV than those in cluster I, their mean hair Ca levels and serum OC and CTx concentrations did not differ significantly. The explanation may be in phenomena related to calcium absorption or those related to its urinary excretion.

Absorption is a crucial regulator of $\mathrm{Ca}$ homeostasis in human body. It increases during periods of high physiologic requirements or those in which the Ca intake is lower [20, 24]. It has been reported that significant increases in $\mathrm{Ca}$ intake did not result in better $\mathrm{Ca}$ balance but to a significant increase in fecal $\mathrm{Ca}$ excretion in young adults [25]. So it may be assumed that there is a higher percentage of $\mathrm{Ca}$ absorption in women with lower $\mathrm{Ca}$ consumption, which would at least in part explain the situation for clusters I and IV. It is also possible that the presence of $\mathrm{Ca}$ absorption inhibitors such as oxalates, phytates and fiber play a relevant role [26]. In cluster IV the intake of fiber was higher than in cluster I, but it was not above dietary recommended values, so it might have a minor impact on $\mathrm{Ca}$ absorption. The intake of oxalates and phytates was not evaluated in the present study. 
A number of factors increase urinary $\mathrm{Ca}$ excretion: high intake of sodium, protein, caffeine, diuretics or a high dietary acid load [27, 28]. It is possible that women in cluster I might have lower urinary $\mathrm{Ca}$ excretion than those in cluster IV as they consumed significantly less protein, coffee and possibly less total $\mathrm{Na}$, although dietary $\mathrm{Na}$ was comparable in both clusters.

The latter can be presumed on the fact that even though the $\mathrm{Na}$ amount derived from table salt was not calculated, salt could be an important source in cluster IV, where food consumption was significantly higher than in group I $(p<0.05$, data not shown) with consequent higher intake of salt, as customary in Poland. A study conducted among Polish students showed the daily consumption of salt is $4-9$ g per person per day [29].

On the other hand, compared with cluster IV the diet of women in cluster I contained almost two times the amount of meat products, which imposes a high acid load, thus increasing hypercalciuria. With exception of milk and dairy products, there were no significant differences in other food groups that may play a role in acid-base homeostasis in the body. The influence of dairy products may be twofold. Milk and yogurt are alkaline and cheeses are acidic, so nothing can be said about their influence on renal acid load.

Another issue is the significantly lower $\mathrm{Ca} / \mathrm{P}$ intake ratio observed in cluster I. This imbalance between dietary calcium and phosphorus intake can adversely affect the $\mathrm{Ca}$ metabolism and bone health [30].

The very high mean hair Ca level in cluster II may be due to a lower rate of hair growth resulting in higher accumulation of minerals. Such situation has been reported for $\mathrm{Zn}, \mathrm{Fe}$, and $\mathrm{Cu}$ in malnourished children [31,32]. Although women in cluster II were rather "small eaters", they were not malnourished. With exception of meat products they consumed similar amounts of almost all other food groups, macro- and micronutrients (beside vitamin PP) than cluster I. Above reference values of hair Ca level in women from cluster II could not be explained by their nutritional parameters or life-style factors considered in this work and requires additional study. It is also worth noticing that although they had the highest mean hair $\mathrm{Ca}$ level, they also had the lowest mean serum $\mathrm{Ca}$ concentration.

Elevated $\mathrm{Ca}$ hair levels are mainly related to some pathologic conditions. Results from fibromyalgia patients and conclusions from a study on rats and monkeys [8], allowed the author to conclude that high levels of $\mathrm{Ca}$ and $\mathrm{Mg}$ in hair were related to a decreased body pool. Also, very high hair $\mathrm{Ca}$ concentrations $(>1,900 \mu \mathrm{g} / \mathrm{g})$ in women with primary hyperparathyroidism or hyperthyroidism, suggested that hair acts as a minor excretory organ, reflecting well the increased Ca mobilization from the bone in both pathologies [5]. This could not be the case in our study as the bone biomarkers in clusters were similar.

Young women participated in this study did not suffer from chronic diseases and were not on medication that might affect $\mathrm{Ca}$ metabolism. Also, there were no significant differences in self-perceived physical activity, use of contraceptives or smoking status, so these factors are not expected to influence Ca status/utilization among clusters. Moreover, hair treatments like dyes or permanent wave may increase the level of minerals in this material [4] but our volunteers declared not to use such treatments for at least three past months.

In this study, no significant correlations between hair $\mathrm{Ca}$ levels and other measured variables: Ca or other nutrients intake, biochemical and somatic parameters were seen in the entire group. In cluster IV the women had a well-balanced diet, but too low vitamin D intake there was an inverse correlation between hair $\mathrm{Ca}$ and vitamin $\mathrm{D}$ intake $r=-0.57 ; p=$ 0.033 . It could be assumed that higher vitamin $\mathrm{D}$ intakes would result in more $\mathrm{Ca}$ been preferably incorporated into bone and not into hair. In the same cluster, the Ca levels in hair and in serum were negatively correlated $(r=-0.56 ; p=0.037)$ as well as with BMI $(r=$ 
$-0.65 ; p=0.012$ ), and higher $\mathrm{Ca}$ serum levels were directly related to higher $\mathrm{CTx}$ serum levels $(r=0.49 ; p=0.072)$. In addition, the concentrations of $\mathrm{Ca}$ and $\mathrm{Mg}$ in hair were also positively correlated $(r=0.57 ; p=0.033)$. These results are in good agreement with observations from other studies [3, 9, 33].

Stepwise multiple regression analysis revealed that data collected in this study do not allow a full explanation of the Ca concentration in hair. It does show, however, that a higher intake of $\mathrm{Mg}$ can result in a higher content of $\mathrm{Ca}$ in hair in young women. This can be explained by the synergistic action of those minerals in the body. Probably, the enzyme involved in the activation process of vitamin D renal hydroxylation step is magnesiumdependent. On the other hand, the relationship between $\mathrm{Ca}$ and $\mathrm{Mg}$ might have an antagonistic character as they compete for intestinal absorption [34].

The bone mineral density, PTH, serum vitamin D and urine Ca excretion were not included in this study. These parameters may help to account for the observed broad range of $\mathrm{Ca}$ levels in the hair of healthy young women.

\section{Conclusions}

At adequate $\mathrm{Ca}$ intake and comparable serum bone biomarker levels, higher vitamin $\mathrm{D}$ intake from supplements positively correlated to higher $\mathrm{Ca}$ deposition in hair. At much lower than recommended vitamin $\mathrm{D}$ intake and similar serum bone biomarker levels, hair $\mathrm{Ca}$ concentrations were similar in women consuming low amounts of $\mathrm{Ca}$ but high amounts of meat products and in women whose diets were rich in dairy products and Ca. Probably, the homeostatic mechanisms operated and in consequence, comparable $\mathrm{Ca}$ amounts were retained in the body. Above reference values of hair Ca levels in $25 \%$ of subjects could not be explained by their nutritional habits or life-style factors analyzed in the study. Although our results showed that hair $\mathrm{Ca}$ concentrations were weakly related to the quality of the diet, some synergistic interactions between nutrients, especially with vitamin D and Mg were observed.

Acknowledgments This work was partly funded by a grant from the Ministry of Science and Higher Education (MNiSzW), Poland. We thank the Nordic Bioscience Diagnostics for their donation of Oc and CTx ELISA kits.

Open Access This article is distributed under the terms of the Creative Commons Attribution Noncommercial License which permits any noncommercial use, distribution, and reproduction in any medium, provided the original author(s) and source are credited.

\section{References}

1. Hambidge KM (1982) Hair analyses: worthless for vitamins, limited for minerals. Am J Clin Nutr 36:943-949

2. Attar KM, Abdel-Aal MA, Debayle P (1990) Distribution of trace elements in the lipid and nonlipid matter of hair. Clin Chem 36:477-480

3. Dunicz-Sokołowska A, Długaszek M, Radomska K et al (2007) Contents of bioelements and toxic metals in the Polish population determined by hair analysis. Part III. Adults aged 20 to 40 years. Magnes Res 20:43-52

4. Radomska K, Graczyk A, Konarski J (1993) Contents of macro- and microelements in human body determined by hair analysis. Population studies. Clin Chem Enzymol Commun 5:105-118 
5. Miekeley N, de Carvalho Fortes LM, Porto da Silveira CL et al (2001) Elemental anomalies in hair as indicators of endocrinologic pathologies and deficiencies in calcium and bone metabolism. J Trace Elem Med Biol 15:46-55

6. MacPherson A, Bacsó J (2000) Relationship of hair calcium concentration to incidence of coronary heart disease. Sci Total Environ 255:11-19

7. Song CH, Barrett-Connor E, Chung JH, Kim SH, Kim KS (2007) Associations of calcium and magnesium in serum and hair with bone mineral density in premenopausal women. Biol Trace Elem Res 118:1-9

8. Yan S (1999) Hair calcium and magnesium levels in patients with fibromyalgia: a case center study. J Manipulative Physiol Ther 22:586-593

9. Wang CT, Chang WT, Zeng WF et al (2005) Concentrations of calcium, copper, iron, magnesium, potassium, sodium and zinc in adult female hair with different body mass indexes in Taiwan. Clin Chem Lab Med 43:389-393

10. Lubkowska A, Sobieraj W (2009) Concentrations of magnesium, calcium, iron, selenium, zinc and copper in the hair of autistic children. Trace Elem Electrolytes 26:72-77

11. Tsukada N, Sugahara A (1996) Study on trace element values in hair analysis of boys living in national home for resocialization of minors. Jpn J Nutr 54:33-40 (English abstract)

12. Kałuża J, Brzozowska A (2005) Realization of nutritional recommendations and mineral and trace element levels in serum and hair of elderly from Warsaw region. Żyw Człow Metab 32(suppl No 1, part II):765-770 (English abstract)

13. Gonzalez-Reimers E, Martin-González MC, Galindo-Martin L et al (2008) Hair zinc, copper and iron relationships with quality of diet, tobacco smoking and nutritional status. Trace Elem Electrolytes 25:3540

14. Szponar L, Wolnicka K, Rychlik E (2000) Album of photographs of food products and dishes. National Food and Nutrition Institute, Warsaw

15. Jeruszka-Bielak M, Pietruszka B (2006) Photo album of fortified foods and supplements available on Polish market in 2005-2006. Warsaw, Poland

16. Zachwieja Z (1999) Conference of Polish Academy of Science: noninvasive methods for assessing macro- and microelement status, Warsaw, Poland

17. Institute of Medicine (IOM), DRI-Dietary Reference Intakes (2010) Dietary reference intakes for calcium and vitamin D, Report brief. Available at: http://www.iom.edu/Reports/2010/Dietary-Reference-Intakesfor-Calcium-and-Vitamin-D.aspx. Accessed 4 Jan 2011

18. DRI-Dietary Reference Intakes (Normy Żywienia Człowieka) (2008) Jarosz M, Bułhak-Jachymczyk B (eds) PZWL, Warsaw, Poland

19. Kabata J, Kalinowski L, Szczepańska-Konkel M et al (2005) Laboratory analysis in everyday practicereference values and interpretations. MAKmedia, Gdańsk

20. Fishbein L (2004) Multiple sources of dietary calcium — some aspects of its essentiality. Regul Toxicol Pharmacol 39:67-80

21. MacPherson A, Balint J, Bacsó J (1995) Beard calcium concentration as a marker for coronary heart disease as affected by supplementation with micronutrients including selenium. Analyst 120:871-875

22. Reid I (2003) Vitamin D and fracture prevention. In: New SA, Bonjour J-P (eds) Nutritional aspects of bone health. RSC, Cornwall, pp 175-191

23. Gundberg CM, Looker AC, Nieman SD et al (2002) Patterns of osteocalcin and bone specific alkaline phosphatase by age, gender, and race or ethnicity. Bone 31:703-708

24. Fairweather-Tait SJ, Teucher B (2002) Iron and calcium bioavailability of fortified foods and dietary supplements. Nutr Rev 60:360-367

25. Kohlenberg-Mueller K, Raschka L (2003) Calcium balance in young adults on a vegan and lactovegetarian diet. J Bone Miner Metab 21:28-33

26. Pettifor J (2003) Rickets, osteomalacia and other metabolic bone diseases: influence of nutrition. In: New SA, Bonjour J-P (eds) Nutritional aspects of bone health. RSC, Cornwall, pp 65-90

27. Lin PH, Ginty F, Appel L et al (2003) The DASH diet and sodium reduction improve markers of bone turnover and calcium metabolism in adults. J Nutr 133:3130-3136

28. Wigertz K, Palacios C, Jackman LA et al (2005) Racial differences in calcium retention in response to dietary salt in adolescent girls. Am J Clin Nutr 81:845-850

29. Marzec Z, Marzec A, Zaręba S (2004) Evaluation of sodium, calcium, magnesium, iron and copper intake with students daily food rations. International Scientific Conference: Physiological determinants of progress in diet therapy. SGGW, Warsaw, pp 604-608

30. Calvo M, Carpenter T (2003) The influence of phosphorus on the skeleton. In: New SA, Bonjour J-P (eds) Nutritional aspects of bone health. RSC, Cornwall, pp 229-265

31. Erten J, Arcasoy A, Cavdar AO et al (1978) Hair zinc levels in healthy and malnourished children. Am J Clin Nutr 31:1172-1174 
32. Weber CW, Nelson GW, Vasquez de Vaquera M et al (1990) Trace elements in the hair of healthy and malnourished children. J Trop Pediatr 36:230-234

33. Khalique A, Ahmad S, Anjum T et al (2005) A comparative study based on gender and age dependence of selected metals in scalp hair. Environ Monit Assess 104:45-57

34. Toba Y, Masuyama R, Kato K et al (1999) Effects of dietary magnesium level on calcium absorption in growing male rats. Nutr Res 19:783-793 